Pacific Journal of Mathematics

ALGEBRAICALLY DEFINED SUBSPACES IN THE
COHOMOLOGY OF A KUGA FIBER VARIETY 


\section{ALGEBRAICALLY DEFINED SUBSPACES IN THE COHOMOLOGY OF A KUGA FIBER VARIETY}

\section{B. BRENT GORDON}

Let $G$ be a semisimple algebraic group of hermitian type defined over $\mathbf{Q}$, let $\mathfrak{X} \simeq G_{\mathbf{R}} / K$, where $K \subset G$ is a maximal compact subgroup, be the symmetric domain associated to $G$, let $\Gamma$ be an arithmetic subgroup of $G$, let $(\pi, E)$ be a finite-dimensional representation of $G$ defined over $Q$, let $\mathscr{U}:=\Gamma \backslash \mathfrak{X}$, and let $\mathscr{E}$ be the locally constant sheaf over $\mathscr{U}$ associated to $(\pi, E)$. Then under certain conditions on $G, \Gamma$ and $(\pi, E)$, the quotient $\mathscr{U}$ is a complex projective variety and there exists a Kuga fiber variety $\mathscr{V}$, i.e., a complex projective variety with the structure of an analytic family of abelian varieties parametrized by $\mathscr{U}$, such that $H^{a}(\mathscr{U} ; \mathscr{E})$ may be identified with a subspace of $H^{*}(\mathscr{V} ; \mathbf{Q})$. The purpose of this paper is to show that for a certain class of nontrivial $(\pi, E)$ the subspace of $H^{*}(\mathscr{V} ; \mathbf{Q})$ with which $H^{a}(\mathscr{U} ; \mathscr{E})$ is identified is algebraically defined, or in other words that this subspace is contained in $H^{r}(\mathscr{V} ; \mathbf{Q})$ for some $r$ and a projection from $H^{r}(\mathscr{V} ; \mathbf{Q})$ to it is induced by an algebraic class in $H^{*}(\mathscr{V} \times \mathscr{V} ; \mathbf{Q})$. In particular, since the projection of an algebraic class in $H^{r}(\mathscr{V} ; \mathbf{Q})$ is again an algebraic class, this paper provides an answer to the question of how to define algebraic classes in $H^{a}(\mathscr{U} ; \mathscr{E})$ for some nontrivial local coefficient systems $\mathscr{E}$.

Introduction. Let $G$ be a semisimple algebraic group defined over $\mathbf{Q}$, of hermitian type and Q-rank zero, and let $\Gamma$ be a torsion-free arithmetic subgroup of $G$ which is Zariski dense in $G$ even when $G_{\mathbf{R}}$ has compact factors [1] and contained in the identity component of $G_{\mathbf{R}}$. Further, let $\mathfrak{X} \simeq G_{\mathrm{R}} / K$, for some maximal compact $K \subset G_{\mathrm{R}}$, be the symmetric domain associated to $G$, and let $(\sigma, W, \beta)$ be a symplectic representation of $G$ defined over Q. In 1963 Kuga [12] showed how to associate to $(G, \Gamma, \mathfrak{X},(\sigma, W, \beta))$ together with some additional data an analytic family $\mathscr{V}$ of abelian varieties parametrized by the locally symmetric variety $\mathscr{U}:=\Gamma \backslash \mathfrak{X}$ such that if $(\sigma, W, \beta)$ satisfies a certain analyticity condition (condition $\left(H_{1}\right)$, cf. paragraph 1A below) then $\mathscr{V}$ is a complex projective variety. Such a variety $\mathscr{V}$ is called a Kuga fiber variety; cf. paragraph $1 \mathrm{C}$ below for more details.

One of Kuga's many motivations for studying Kuga fiber varieties was the observation that they provide a natural algebraic-geometric realization for the cohomology of arithmetic groups with nontrivial coefficients. That is, suppose $(\pi, E)$ is a representation of $G$ defined over $\mathbf{Q}$ 
which is contained in some exterior power $\Lambda^{b}(\sigma, W)$ of $(\sigma, W)$. Then there is an embedding of $H^{a}(\Gamma ; E)$ into $H^{a+b}(\mathscr{V} ; \mathbf{Q})$ which depends only on a choice of base point in $\mathfrak{X}$ (Lemma 1D). We call the image of $H^{a}(\Gamma ; E)$ in $H^{a+b}(\mathscr{V} ; \mathbf{Q})$ the representation-theoretic component of $H^{*}(\mathscr{V} ; \mathbf{Q})$ corresponding to $H^{a}(\Gamma ; E)$. Since $H^{a}(\Gamma ; E)$ is isomorphic to $H^{a}(\mathscr{U} ; \mathscr{E})$ where $\mathscr{E}$ is the locally constant sheaf on $\mathscr{U}$ associated to $(\pi, E)$, the same representation-theoretic component of $H^{*}(\mathscr{V} ; \mathbf{Q})$ also corresponds to this space; however, it is just a little bit simpler to state our results in terms of $H^{a}(\Gamma ; E)$.

The purpose of this paper is to prove the following theorem.

THEOREM 1. Let $G$ be a semisimple algebraic group defined over $\mathbf{Q}$, of hermitian type and Q-rank zero, let $\Gamma$ be a Zariski-dense arithmetic subgroup of $G$ which is torsion-free and contained in the identity component of $G_{\mathbf{R}}$, and suppose $(\pi, E)$ is a $\mathbf{Q}$-privileged representation of $G$. Then there exists a Kuga fiber variety $\mathscr{V}$ such that the representation-theoretic component of $H^{*}(\mathscr{V} ; \mathbf{Q})$ corresponding to $H^{a}(\Gamma ; E)$ is an algebraically defined subspace of $H^{*}(\mathscr{V} ; \mathbf{Q})$.

Some explanations are in order. Firstly, in general a subspace $H^{r}(\mathscr{M} ; \mathbf{Q})$ of the rational cohomology $H^{*}(\mathscr{A} ; \mathbf{Q})$ of a complex projective variety $\mathscr{A}$ is said to be algebraically defined if $H^{r}(\mathscr{M} ; \mathbf{Q}) \subset H^{r}(\mathscr{A} ; \mathbf{Q})$ for some $r$ and there is a projection from $H^{r}(\mathscr{A} ; \mathbf{Q})$ to $H^{r}(\mathscr{M} ; \mathbf{Q})$ induced by an algebraic class in $H^{*}(\mathscr{A} \times \mathscr{A} ; \mathbf{Q})$; cf. paragraph $1 \mathrm{E}$ for more detail. Philosophically the distinction between an algebraically defined subspace of the rational cohomology of a complex projective variety and the Betti realization of a motive $[2, \S 0 ; 8 ; 14]$ is quite small, but it does depend on a "standard conjecture" of Grothendieck [7] not yet proved; again, cf. paragraph 1E. In any case, if $H^{r}(\mathscr{M} ; \mathbf{Q}) \subset H^{r}(\mathscr{A} ; \mathbf{Q})$ is an algebraically defined subspace, then algebraic classes in $H^{r}(\mathscr{A} ; \mathbf{Q})$ project to algebraic classes in $H^{r}(\mathscr{M} ; \mathbf{Q})$. Thus, remembering that $\mathscr{U}:=$ $\Gamma \backslash \mathfrak{X}$ is also a complex projective variety [8], Theorem 1 provides, in certain cases, an answer to the question of how to define algebraic classes in $H^{a}(\mathscr{U} ; \mathscr{E})$ (or $H^{a}(\Gamma ; E)$ ) when $\mathscr{E}$ is a nontrivial local coefficient system associated to a representation $(\pi ; E)$ of $G$.

Secondly, we say that a representation $(\pi, E)$ of $G$ defined over $\mathbf{Q}$ is privileged over $\mathbf{Q}$, or $\mathbf{Q}$-privileged, if (a) there exists a symplectic representation $(\sigma, W, \beta)$ of $G$ defined over $\mathbf{Q}$ which satisfies the analyticity condition $\left(H_{1}\right)$ (cf. paragraph $\left.1 \mathrm{~A}\right)$ such that $(\pi, E)$ is contained in some exterior power $\wedge^{b}(\sigma, W)$ of $(\sigma, W)$, and (b) the projection from $\wedge^{b} W$ to $E$ satisfies a certain condition, which in case $G_{\mathbf{R}}$ has no compact factors is 
that the idempotent inducing this projection should belong to the subalgebra of End ${ }_{\mathbf{Q}}\left(\wedge^{b} W\right)$ generated by the natural image therein of End ${ }_{G}(W)$, where $\operatorname{End}_{G}(W)$ is the commutator algebra of $\sigma(G)$ in $\operatorname{End}_{\mathbf{Q}}(W)$. For more details, cf. paragraph 1B. Some examples of privileged representations are discussed in paragraph 3B; we mention here only that if $G$ is a Q-simple Q-form of a classical group, such as a unitary or symplectic group, and $G_{\mathbf{R}}$ has no compact factors, then every Q-primary representation of $G$ is also privileged over $\mathbf{Q}$.

Thirdly, we remark that the requirement that $\Gamma$ be torsion-free and contained in the connected component of $G_{\mathbf{R}}$ should be thought of as more technical than substantive, since any (Zariski-dense) arithmetic subgroup of $G$ will contain a normal subgroup of finite index satisfying these conditions [21, Lemma IV.7.2]. We also observe that much of the theory of the present paper can be carried over to the case where $G$ has Q-rank greater than zero. In this case $\mathscr{U}$ and $\mathscr{V}$, as defined here, are noncompact quasiprojective varieties, so it would be necessary to restrict attention to the compactly supported cohomology. Alternatively, sometimes it is reasonable to compactify $\mathscr{U}$ and $\mathscr{V}$ and then work with the relative cohomology. However, we will not pursue these ideas any further in the present work, though we hope to return to them sometime in the future.

Finally, regarding the proof of Theorem 1 there are two key points to be noted. Let $\mathscr{F}$ be a generic abelian variety in the family $\mathscr{V}$. Then under the natural identification of $H^{*}(\mathscr{F} ; \mathbf{Q})$ with $\Lambda^{*}\left(W_{\mathbf{Q}}\right)$ (cf. (5)), the condition that $(\pi, E)$ be a $\mathbf{Q}$-privileged representation of $G$ contained in $\wedge^{b} W$, together with a proposition of $\operatorname{Kuga}[13, \S \mathrm{F}]$ (cf. (3)) about the endomorphisms of $\mathscr{F}$, implies that $E$ is an algebraically defined subspace of $H^{*}(\mathscr{F} ; \mathbf{Q})$ such that a projection from $H^{b}(\mathscr{F} ; \mathbf{Q})$ to $E$ can be induced by some combination of (the graphs of) endomorphisms of $\mathscr{F}$. The second key point, embodied in Lemma $2 \mathrm{~B}$, is that an endomorphism of $\mathscr{F}$ can be extended in a natural way to an endomorphism of $\mathscr{V}$, so that the extension of whatever combination of endomorphisms of $\mathscr{F}$ induces a projection from $H^{b}(\mathscr{F} ; \mathbf{Q})$ to $E$ can be used to induce a projection from $H^{a+b}(\mathscr{V} ; \mathbf{Q})$ to the subspace corresponding to $H^{a}(\Gamma ; E)$, showing that this subspace is algebraically defined.

In order to get to the proof of Theorem 1 as quickly as possible, the necessary definitions are collected together in $\$ 1$ and discussion of examples is postponed until $\S$. The proof of the theorem occupies $\$ 2$.

Acknowledgments. The author would like to express his most heartfelt thanks to Professor G. Harder for suggesting that he look for a theorem 
like Theorem 1 in a special case. He also wishes to thank Professor S. Kudla for some suggestions for simplifying the proof of that theorem.

1. Definitions. In this section $G$ is a semisimple algebraic group defined over $\mathbf{Q}$ and $\mathfrak{X} \simeq G_{\mathbf{R}} / K$, for a maximal compact subgroup $K \subset G_{\mathbf{R}}$, is the symmetric space associated to $G$.

A. Condition $\left(H_{1}\right)$. (Satake $[19 ; 20 ; 21]$.) Let $(\sigma, W, \beta)$ be a symplectic representation of $G$ defined over $\mathbf{Q}$, consisting of an even-dimensional vector space $W$, a nondegenerate alternating bilinear form $\beta$ on $W \times W$ and a homomorphism $\sigma: G \rightarrow \operatorname{Sp}(W, \beta)$, where

$$
\operatorname{Sp}(W, \beta):=\{g \in \mathrm{GL}(W) \mid \beta(g u, g v)=\beta(u, v) \text { for all } u, v \in W\},
$$

all defined over $\mathbf{Q}$. The map $w \mapsto \beta(w, \cdot)$ gives an isomorphism of $W$ onto its dual $W^{*}$, inducing an isomorphism from $\operatorname{End}(W)$ to $\operatorname{End}\left(W^{*}\right)$ and an identification of $(\sigma, W)$ with its contragredient $\left(\sigma^{*}, W^{*}\right)$, as a representation of $G$. Associated to the symplectic space $(W, \beta)$ is the Siegel space

$$
\begin{array}{r}
\mathfrak{S}(W, \beta):=\left\{J \in \mathrm{GL}\left(W_{\mathbf{R}}\right) \mid J^{2}=-1, \beta(u, J v)=\beta(v, J u),\right. \\
\left.\beta(w, J w)>0 \text { for } u, v, w \in W_{\mathbf{R}}, w \neq 0\right\}
\end{array}
$$

of complex structures on $W_{\mathbf{R}}$, on which $\operatorname{Sp}\left(W_{\mathbf{R}}, \beta\right)$ acts transitively by $J \mapsto g J g^{-1}$. It follows that $\mathfrak{S}(W, \beta) \simeq \mathrm{Sp}\left(W_{\mathbf{R}}, \beta\right) / K_{1}$, for any maximal compact subgroup $K_{1} \subset \operatorname{Sp}\left(W_{\mathbf{R}}, \beta\right)$, can be given the structure of a hermitian symmetric domain associated to $\operatorname{Sp}(W, \beta)$.

Now, if we fix maximal compacts $K \subset G_{\mathbf{R}}$ and $K_{1} \subset \operatorname{Sp}\left(W_{\mathbf{R}}, \beta\right)$ such that $\sigma(K) \subset K_{1}$, then $\sigma$ induces a map $\tau: \mathfrak{X} \rightarrow \mathfrak{S}(W, \beta)$, called the Eichler map [12, §II.6], such that the diagram

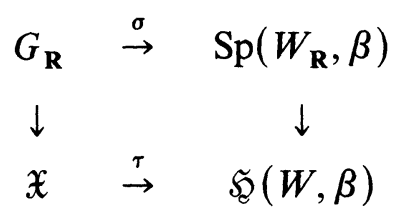

commutes. When we wish to think of the Eichler map as associating a complex structure on $W_{\mathbf{R}}$ to a point $x \in \mathfrak{X}$ we may write $J_{x}$ for $\tau(x)$.

Definition. When $G$ is of hermitian type, so that $\mathfrak{X}$ may be given the structure of a hermitian domain, then a symplectic representation $(\sigma, W, \beta)$ of $G$ defined over $\mathbf{Q}$ is said to satisfy condition $\left(H_{1}\right)$ iff $\sigma$ preserves the Cartan decomposition (so that the Eichler map induced by $\sigma$ is holomorphic). 
Satake has made a study of which semisimple algebraic groups over $\mathbf{Q}$ have symplectic representations which satisfy condition $\left(H_{1}\right)$ and what some of those representations are; see paragraph $3 \mathrm{~A}$ below, or $[\mathbf{2 0} ; \mathbf{2 1}]$.

B. Privileged representations. Given a symplectic representation $(\sigma, W, \beta)$ of $G$, let

$$
\begin{array}{r}
C_{1}(G, W):=\{\alpha \in \operatorname{End}(W) \mid \alpha \sigma(g)=\sigma(g) \alpha \text { for all } g \in G, \text { and } \\
\qquad \alpha J=J \alpha \text { for all } J \in \tau(\mathfrak{X})\},
\end{array}
$$

and let $C_{b}(G, W)$ be the subalgebra of $\operatorname{End}\left(\wedge^{b} W\right)$ generated by the image of $C_{1}(G, W)$ under the natural map $\operatorname{End}(W) \rightarrow \operatorname{End}\left(\wedge^{b} W\right)$, where $\wedge^{b} W$ is the $b$ th exterior power of $W$. In case $\tau(\mathfrak{X})$ is contained in the linear closure of $\sigma\left(G_{\mathbf{R}}\right)$, which will be the case if $G_{\mathbf{R}}$ has no compact factors [13, $\S \mathrm{F}]$, then $C_{1}(G, W)$ is precisely the commutator algebra of $\sigma(G)$ in End $(W)$. However, even when $C_{1}(G, W)$ is the commutator algebra of $\sigma(G)$ in $\operatorname{End}(W)$, in general $C_{b}(G, W)$, for $b>1$, will be much smaller than the commutator algebra of $\left(\wedge^{b} \sigma\right)(G)$ in $\operatorname{End}\left(\wedge^{b} W\right)$.

The following class of representations will be of special interest to us.

Definition. A representation $(\pi, E)$ of $G$ will be said to be privileged iff (i) there exists a symplectic representation $(\sigma, W, \beta)$ of $G$ which satisfies condition $\left(H_{1}\right)$ and a nonnegative integer $b$ such that $(\pi, E)$ is contained in $\wedge^{b}(\sigma, W)$, and (ii) the projection $P: \wedge^{b} W \rightarrow E$ is an element of $C_{b}(G, W)$. If $E$ and $P$ are defined over a field $F$ containing $\mathbf{Q}$, then we may say that $(\pi, E)$ is privileged over $F$, or $F$-privileged.

For example, if $T$ is an element of $C_{b}(G, W)_{F}$ and $F$ contains all the eigenvalues of $T$, then every characteristic subspace of $T$ is privileged over $F$. For the projection $P: \wedge^{b} W \rightarrow E$ from $\wedge^{b} W$ to a characteristic subspace $E$ of $T$ is a polynomial in $T$ with coefficients in $F$ [17, Thm. 6.1], hence $P$ is also in $C_{b}(G, W)$. In particular, we deduce the following.

LEMMA. If $W \simeq \oplus_{i=1}^{r} W_{i}$ as a $G$-module over $F$, then

$$
\wedge^{b} W \simeq \bigoplus_{\substack{\left(b_{1}, \ldots, b_{r}\right) \in \mathbf{N}^{r} \\ b_{1}+\cdots+b_{r}=b}} \wedge^{b_{1}} W_{1} \otimes \cdots \otimes \wedge^{b_{r}} W_{r}
$$

as a G-module over $F$, and each term on the right-hand side of (1) is privileged over $F$.

Proof. The decomposition (1) is clear, since the exterior product of two disjoint $G$-(sub)modules is isomorphic to their tensor product. Then the terms on the right-hand side of (1) are characterized as the $\Pi p_{i}^{b_{i} \text {-eigen- }}$ spaces of the element of $\operatorname{End}\left(\wedge^{b} W\right)$ which comes from the element of 
$C_{1}(G, W)$ which acts as multiplication by $p_{i}$ on $W_{i}$, where $p_{1}, \ldots, p_{r}$ are any $r$ distinct primes. The lemma now follows from the remarks above.

Further examples of privileged representations will be given in paragraph 3B, below.

C. Group-theoretic families of abelian varieties. (Kuga [12], or cf. [21, Ch. IV].) Now let $\Gamma \subset G$ be a torsion-free arithmetic subgroup which we assume to be Zariski-dense in $G$ even if $G_{\mathbf{R}}$ has compact factors [1]. Let $\Lambda \subset W$ be a $\mathbf{Z}$-lattice of maximal rank such that

$$
\sigma(\Gamma) \Lambda=\Lambda \text { and } \beta(\Lambda, \Lambda) \subset \mathbf{Z} \text {. }
$$

Then the quotient $\mathscr{V}:=\Gamma \backslash\left(\mathfrak{X} \times\left(W_{\mathbf{R}} / \Lambda\right)\right)$, which is well-defined by (2), is a $C^{\infty}$-torus bundle over $\mathscr{U}:=\Gamma \backslash \mathfrak{X}$, where the projection $\varphi: \mathscr{V} \rightarrow \mathscr{U}$ is induced by the natural projection $\mathfrak{X} \times\left(W_{\mathbf{R}} / \Lambda\right) \rightarrow \mathfrak{X}$. Moreover, the fibers of $\varphi$ can be given the structure of polarized abelian varieties: For when $F_{x}:=x \times W_{\mathbf{R}} / \Lambda$, with $x \in \mathfrak{X}$, is endowed with the complex structure $J_{x}$ and the polarization $\beta$, it follows from (2) that the abelian variety $\left(F_{x}, J_{x}, \beta\right)$ is isomorphic to $\left(F_{\gamma(x)}, J_{\gamma(x)}, \beta\right)$. Then $\varphi^{-1}(u)$ may be unambiguously identified with $\left(F_{x}, J_{x}, \beta\right)$ whenever $x \in \mathfrak{X}$ is a preimage of $u \in \mathscr{U}$, and $\mathscr{V}$ may be called a group-theoretic family of abelian varieties.

If, in addition, $G$ is of Q-rank zero, then $\mathscr{U}$ and $\mathscr{V}$ are compact ([2] or [18]), and if furthermore $G$ is of hermitian type, then $\mathscr{U}$ can be embedded as a complex projective variety [10]. And finally, if $(\sigma, W, \beta)$ satisfies condition $\left(H_{1}\right)$ as well, then $\mathscr{V}$, too, can be embedded as a complex projective variety in such a way that $\varphi: \mathscr{V} \rightarrow \mathscr{U}$ is holomorphic ([12], or cf. [21, Ch. IV]). When all these conditions are met, the grouptheoretic family of abelian varieties $\mathscr{V}$ is called a Kuga fiber variety. Various properties of Kuga fiber varieties have been studied by Kuga and his students (among others); see [14] and the bibliography there. Here we merely remark that when $u$ is a generic point of $\mathscr{U}$, then there is a natural isomorphism

$$
\operatorname{End}\left(\varphi^{-1}(u)\right) \otimes \mathbf{Q} \cong C_{1}(G, W)_{\mathbf{Q}}
$$

see $[13, \S F]$.

D. Representation-theoretic components. In this paragraph we make some observations about the cohomology of a group-theoretic family of abelian varieties (although everything we will say in this paragraph can be applied to the more general situation of a torus bundle over a locally 
symmetric space). We assume only that $\mathscr{V}$ and $\mathscr{U}$ are compact. Then the following lemma is implicit in [14, (1.3.3)]; however, some comments concerning its proof will help to establish our notation.

Lemma. If $E$ is a G-invariant subspace of $\wedge^{b} W$ defined over $\mathbf{Q}$, for some $b \geq 0$, then there exists an embedding of $H^{a}(\Gamma ; E)$ into $H^{a+b}(\mathscr{V} ; \mathbf{Q})$ which depends only on a choice of base point in $\mathfrak{X}$.

Definition. When $(\pi, E)$ is a representation of $G$ defined over $\mathbf{Q}$ which is contained in $\Lambda^{b}(\sigma, W)$ for some $b \geq 0$, then the image of $H^{a}(\Gamma ; E)$ in $H^{a+b}(\mathscr{V} ; \mathbf{Q})$ will be called the representation-theoretic component of $H^{*}(\mathscr{V} ; \mathbf{Q})$ corresponding to $H^{a}(\Gamma ; E)$.

Proof of lemma (sketch). Given a base point $x \in \mathfrak{X}$, say $x=K$ when $\mathfrak{X} \stackrel{\leftrightarrow}{\leftrightarrow} G_{\mathbf{R}} / K$, with $\Gamma x=: u \in \mathscr{U}$, there is an identification

$$
\pi_{1}(\mathscr{U}, u) \stackrel{\sim}{\leftrightarrow} \Gamma
$$

obtained by letting $\gamma \in \Gamma$ correspond to the class in $\pi_{1}(\mathscr{U}, u)$ of the image in $\mathscr{U}$ of a path in $\mathfrak{X}$ joining $x$ to $\gamma(x)$. Similarly, identifying the underlying topological space of $\mathscr{F}:=\varphi^{-1}(u)$ with $W_{\mathbf{R}} / \Lambda$ and taking the origin of $W_{\mathbf{R}}$ as a base point, $H_{1}(\mathscr{F} ; \mathbf{Z})$ may be identified with $\Lambda$. Then $H_{1}(\mathscr{F} ; \mathbf{Q})$ is identified with $\Lambda \otimes \mathbf{Q}=W_{\mathbf{Q}}$, and

$$
H^{b}(\mathscr{F} ; \mathbf{Q}) \stackrel{\sim}{\leftrightarrow} \wedge^{b} W_{\mathbf{Q}}
$$

for $b \geq 0$, recalling from paragraph $1 \mathrm{~A}$ that $W^{*}$ may be identified with $W$. With these identifications, the action of $\pi_{1}(\mathscr{U}, u)$ on $H^{b}(\mathscr{F} ; \mathbf{Q})$ coincides with the $\wedge^{b} \sigma$-action of $\Gamma$ on $\wedge^{b} W_{\mathbf{Q}}$. Moreover, since $\mathfrak{X}$ is simply connected, $\mathscr{U}$ is an Eilenberg-Mac Lane $K(\Gamma, 1)$-space so

$$
H^{a}\left(\Gamma ; \wedge^{b} W\right) \simeq H^{a}\left(\mathscr{U} ; R^{b} \varphi_{*}(\mathbf{Q})\right)
$$

where $R^{b} \varphi_{*}(\mathbf{Q})$ is the locally constant sheaf over $\mathscr{U}$ associated to the action of $\pi_{1}(\mathscr{U}, u)$ on $H^{b}(\mathscr{F} ; \mathbf{Q})$.

As the inclusion of $E$ in $\wedge^{b} W$ induces an inclusion of $H^{a}(\Gamma ; E)$ into $H^{a}\left(\Gamma ; \wedge^{b} W\right)$, to prove the lemma it will suffice to find a subspace of $H^{a+b}(\mathscr{V} ; \mathbf{Q})$ which is naturally isomorphic to $H^{a}\left(\mathscr{U} ; R^{b} \varphi_{*}(\mathbf{Q})\right)$. For $m \in \mathbf{Z}$, let $\theta_{m}: \mathscr{V} \rightarrow \mathscr{V}$ be the "stretching operator" $[14, \S 1.2]$ induced by the map $(x, w) \mapsto(x ; m w)$ on $\mathfrak{X} \times\left(W_{\mathbf{R}} / \Lambda\right)$. Then $H^{r}(\mathscr{V} ; \mathbf{Q})$ decomposes into a direct sum

$$
H^{r}(\mathscr{V} ; \mathbf{Q})=\bigoplus_{a+b=r} H^{\langle a, b\rangle}(\mathscr{V} ; \mathbf{Q})
$$


of the simultaneous eigenspaces

$$
H^{\langle a, b\rangle}(\mathscr{V} ; \mathbf{Q}):=\left\{\xi \in H^{a+b}(\mathscr{V} ; \mathbf{Q}) \mid \boldsymbol{\theta}_{m}^{*} \xi=m^{b} \text { for all } m \in \mathbf{Z}\right\}
$$

$[14, \S 1.3]$. In addition, the stretching operators $\theta_{m}$ commute with the differentials $d_{s}$, for $s \geq 2$, of the Leray spectral sequence for $\varphi: \mathscr{V} \rightarrow \mathscr{U}$, implying that this spectral sequence degenerates at the $E_{2}$-term. But $H^{\langle a, b\rangle}(\mathscr{V} ; \mathbf{Q})$ is naturally isomorphic to the $a$-th graded component of $H^{a+b}(\mathscr{V} ; \mathbf{Q})$ for the fiber weight filtration giving rise to the Leray spectral sequence, and therefore, by the general theory (cf. e.g. $[6, \S 3.5]$ ), it is naturally isomorphic to $E_{2}^{a, b}=H^{a}\left(\mathscr{U} ; R^{b} \varphi_{*}(\mathbf{Q})\right)$. Thus

$$
H^{\langle a, b\rangle}(\mathscr{V} ; \mathbf{Q}) \simeq H^{a}\left(\Gamma ; \wedge^{b} W\right)
$$

and the lemma follows.

\section{E. Algebraically defined subspaces (after Grothendieck [7, p. 196]).}

Definition. When $\mathscr{A}$ is a smooth (complex projective) algebraic variety, a subspace $H^{r}(\mathscr{M} ; \mathbf{Q}) \subset H^{r}(\mathscr{A} ; \mathbf{Q})$, for some $r$, is said to be algebraically defined iff there exists an algebraic cohomology class $\Xi \in$ $H^{*}(\mathscr{A} \times \mathscr{A} ; \mathbf{Q})$ such that a projection $H^{r}(\mathscr{A} ; \mathbf{Q}) \rightarrow H^{r}(\mathscr{M} ; \mathbf{Q})$ is obtained by lifting a class from $H^{r}(\mathscr{A} ; \mathbf{Q})$ to $H^{*}(\mathscr{A} \times \mathscr{A} ; \mathbf{Q})$ via the first projection, cupping with $\Xi$, and taking the image in $H^{r}(\mathscr{A} ; \mathbf{Q})$ under the Gysin homomorphism associated to the second projection.

To describe the nature of $\Xi$ more fully, let $A Z(\mathscr{A} \times \mathscr{A} ; \mathbf{Q})$ denote the Q-algebra generated by the set of all irreducible closed subvarieties in $\mathscr{A} \times \mathscr{A}$ of dimension equal to that of $\mathscr{A}$, where multiplication is defined by $\mathbf{Q}$-linearly extending the usual composition of algebraic correspondences and the diagonal serves as identity. Then the fundamental class map $C \mapsto \operatorname{cl}(C)$, for $C \subset \mathscr{A} \times \mathscr{A}$, extends to a Q-linear map from $A Z(\mathscr{A} \times \mathscr{A} ; \mathbf{Q})$ to $H^{*}(\mathscr{A} \times \mathscr{A} ; \mathbf{Q})$. Thus an algebraic class $\Xi$ inducing a projection $H^{r}(\mathscr{A} ; \mathbf{Q}) \rightarrow H^{r}(\mathscr{M} ; \mathbf{Q})$ as above is the fundamental class of an idempotent in $A Z(\mathscr{A} \times \mathscr{A} ; \mathbf{Q})$. Such a class in $H^{*}(\mathscr{A} \times \mathscr{A} ; \mathbf{Q})$, or any idempotent in $A Z(\mathscr{A} \times \mathscr{A} ; \mathbf{Q})$ representing it, may be referred to as an algebraic projector.

Remark. A subspace $H^{r}(\mathscr{M} ; \mathbf{Q}) \subset H^{*}(\mathscr{A} ; \mathbf{Q})$ is said to be motivic, or more precisely in the context of complex projective varieties, the Betti realization of a motive, if there exists an algebraic projector in $H^{*}(\mathscr{A} \times \mathscr{A} ; \mathbf{Q})$ inducing a projection from $H^{*}(\mathscr{A} ; \mathbf{Q})$ to $H^{r}(\mathscr{M} ; \mathbf{Q})[3$, $\S 0 ; 9 ; 15]$. The obstruction to showing that an algebraically defined subspace is motivic is that it is not known whether $H^{r}(\mathscr{A} ; \mathbf{Q})$, as a subspace of $H^{*}(\mathscr{A} ; \mathbf{Q})$, is motivic. This would follow if the Künneth 
components of the diagonal class in $H^{*}(\mathscr{A} \times \mathscr{A} ; \mathbf{Q})$ were known to be algebraic, which is one of Grothendieck's "standard conjectures" [7].

2. The proof. Let $G$ be a semisimple algebraic group defined over $\mathbf{Q}$, of hermitian type and Q-rank zero, let $\Gamma$ be a Zariski-dense arithmetic subgroup of $G$ which is torsion-free and contained in the connected component of $G_{\mathbf{R}}$, and suppose $(\pi, E)$ is a Q-privileged representation of $G$. Then by assumption there exists a symplectic representation $(\sigma, W, \beta)$ of $G$ defined over $\mathbf{Q}$ which satisfies condition $\left(H_{1}\right)$ such that $(\pi, E)$ is contained in $\wedge^{b}(\sigma, W)$ for some $b \geq 0$. Furthermore, since $\Gamma$ is arithmetic and contained in the identity component of $G_{\mathbf{R}}$, there exists a lattice $\Lambda \subset W$ which satisfies the conditions (2) [21, §IV.7, Remark 2]. Therefore we may associate to the data $(G, \Gamma,(\sigma, W, \beta), \Lambda)$ a Kuga fiber variety $\mathscr{V}$, as in $1 \mathrm{C}$, such that $H^{a+b}(\mathscr{V} ; \mathbf{Q})$ contains a representation-theoretic component corresponding to $H^{a}(\Gamma ; E)$, as in $1 \mathrm{D}$. Now we wish to show that this component is algebraically defined.

A. LEMMA. $H^{\langle a, b\rangle}(\mathscr{V} ; \mathbf{Q})$ is algebraically defined.

Proof. For $m \in \mathbf{Z}$, let $\Theta_{m} \subset \mathscr{V} \times \mathscr{V}$ be the graph of the stretching operator $\theta_{m}$ defined in $1 \mathrm{C}$; in particular $\Theta_{1}$ is the diagonal. Then when $|m|>1$

$$
\Sigma_{m}:=\prod_{0 \leq j \leq \operatorname{dim}_{\mathbf{R}}(\mathscr{U})} \frac{m^{a+b-j} \Theta_{1}-\Theta_{m}}{m^{a+b-j}-m^{b}}
$$

is an algebraic projector which induces a projection from $H^{a+b}(\mathscr{V} ; \mathbf{Q})$ to $H^{\langle a, b\rangle}(\mathscr{V} ; \mathbf{Q})$. For the factor $\left(m^{a+b-j}-m^{b}\right)^{-1}\left(m^{a+b-j} \Theta_{1}-\Theta_{m}\right)$ of $\Sigma_{m}$ kills off the summand $H^{\langle j, a+b-j\rangle}(\mathscr{V} ; \mathbf{Q})$ in the decomposition (7) while acting as the identity on $H^{\langle a, b\rangle}(\mathscr{V} ; \mathbf{Q})$.

B. The key lemma. Now let $\mathscr{F}$ be a generic fiber of $\mathscr{V}$, as in $1 \mathrm{D}$. Then via the identification (5) the representation space $E$ may be identified with a subspace of $H^{b}(\mathscr{F} ; \mathbf{Q})$.

LEMMA. To each $\alpha \in C_{1}(G, W)$ one may associate algebraic cycles $\psi(\alpha) \in A Z(\mathscr{F} \times \mathscr{F} ; \mathbf{Q})$ and $\Psi(\alpha) \in A Z(\mathscr{V} \times \mathscr{V} ; \mathbf{Q})$ in such a way that there exists a Q-algebra isomorphism $\Phi$ from the subalgebra $\mathbf{Q}\left[\psi\left(C_{1}(G, W)\right)\right]$ of $A Z(\mathscr{F} \times \mathscr{F} ; \mathbf{Q})$ generated by the image of $\psi$ onto the subalgebra $\mathbf{Q}\left[\Psi\left(C_{1}(G, W)\right)\right]$ of $A Z(\mathscr{V} \times \mathscr{V} ; \mathbf{Q})$ generated by the image of 
$\Psi$ with the property that $\Psi=\Phi \circ \psi$. Furthermore, if $\mathscr{P} \in \mathbf{Q}\left[\psi\left(C_{1}(G, W)\right)\right]$ is any algebraic projector which induces a projection from $H^{b}(\mathscr{F} ; \mathbf{Q})$ to $E$, then $\Phi(\mathscr{P})$ is also an algebraic projector which induces a projection from $H^{\langle a, b\rangle}(\mathscr{V} ; \mathbf{Q})$ to the representation-theoretic component of $H^{a+b}(\mathscr{V} ; \mathbf{Q})$ corresponding to $H^{a}(\Gamma ; E)$.

Proof. Upon identifying $C_{1}(G, W)$ with $\operatorname{End}(\mathscr{F}) \otimes \mathbf{Q}$ as in (3), we may define $\psi: C_{1}(G, W) \rightarrow A Z(\mathscr{F} \times \mathscr{F}, \mathbf{Q})$ as the $\mathbf{Q}$-linear extension to $C_{1}(G, W)$ of the map that associates to an $\alpha \in \operatorname{End}(\mathscr{F})$ its graph $\psi(\alpha)$ in $\mathscr{F} \times \mathscr{F}$. Similarly $\Psi(\alpha)$ may be defined, for $\alpha \in C_{1}(G, W)$ leaving $\Lambda$ invariant, as the image in $\mathscr{V} \times \mathscr{V}$ of the graph of $(x, w) \mapsto(x, \alpha(w))$, for $(x, w) \in \mathfrak{X} \times W_{\mathbf{R}}$, and extended Q-linearly to all of $C_{1}(G, W)$. Then $\Psi(\alpha)$ is indeed an algebraic cycle, as any $\alpha \in C_{1}(G, W)$ commutes with all $\sigma(\gamma)$ for $\gamma \in \Gamma$ and all $J \in \tau(\mathfrak{X})$. And since $\psi$ (resp. $\Psi$ ) is an isomorphism of $C_{1}(G, W)$ onto its image $\mathbf{Q}\left[\psi\left(C_{1}(G, W)\right)\right]$ (resp. $\mathbf{Q}\left[\Psi\left(C_{1}(G, W)\right)\right]$ ), there does exist a $Q$-algebra isomorphism $\Phi$ such that $\Psi=\Phi \circ \psi$, as indicated in the following diagram.

$$
\begin{array}{ccc}
\operatorname{End}(\mathscr{F}) \otimes \mathbf{Q} & \stackrel{\sim}{\rightarrow} & \mathbf{Q}\left[\psi\left(C_{1}(G, W)\right)\right] \subset A Z(\mathscr{F} \times \mathscr{F}, \mathbf{Q}) \\
1 \downarrow & \psi \nearrow & \downarrow \Phi \\
C_{1}(G, W) & \stackrel{\Psi}{\rightarrow} & A Z(\mathscr{V} \times \mathscr{V}, \mathbf{Q})
\end{array}
$$

Now for $\alpha \in C_{1}(G, W)$ it also follows that $\psi(\alpha)$ induces an endomorphism of $H^{b}(\mathscr{F} ; \mathbf{Q})$ which corresponds via (5) to the endomorphism which $\alpha$ induces on $\Lambda^{b} W$. But then $\Psi(\alpha)$ induces an endomorphism of $H^{\langle a, b\rangle}(\mathscr{V} ; \mathbf{Q})$ which corresponds via (8) to the endomorphism $\alpha$ induces on $H^{a}\left(\Gamma ; \wedge^{b} W\right)$, and the lemma follows.

C. Proof of Theorem 1. Let $\alpha \mapsto \alpha^{\prime}: \operatorname{End}(W) \rightarrow \operatorname{End}\left(\wedge^{b} W\right)$ be the natural map. As $E$ is a privileged constituent of $\wedge^{b} W$, there is a projection $P: \wedge^{b} W \rightarrow E$ of the form $P=p\left(\alpha_{1}^{\prime}, \ldots, \alpha_{r}^{\prime}\right)$ for some polynomial $p$ with coefficients in $\mathbf{Q}$ and some $\alpha_{1}, \ldots, \alpha_{r}$ in $C_{1}(G, W)$. But then $\mathscr{P}:=p\left(\psi\left(\alpha_{1}\right), \ldots, \psi\left(\alpha_{r}\right)\right)$ in $A Z(\mathscr{F} \times \mathscr{F} ; \mathbf{Q})$ is an algebraic projector which induces a projection from $H^{b}(\mathscr{F} ; \mathbf{Q})$ to $E$. Therefore the composition of $\Phi(\mathscr{P})$ with $\Sigma_{m}$ is an algebraic projector in $A Z(\mathscr{V} \times \mathscr{V} ; \mathbf{Q})$ which induces a projection from $H^{a+b}(\mathscr{V} ; \mathbf{Q})$ to the representation-theoretic component corresponding to $H^{a}(\Gamma ; E)$.

3. Examples. When considering the applicability of Theorem 1 two questions immediately arise: Which symplectic representations $(\sigma, W, \beta)$ of what semisimple algebraic groups $G$ defined over $\mathbf{Q}$ satisfy condition $\left(H_{1}\right)$ ? And what are the privileged representations of those groups? 
A. Groups and representations satisfying condition $\left(H_{1}\right)$. Satake [20] has studied the first question and answered it under the additional assumption [20, Part III, eqn. (9)] that the symplectic representation $(\sigma, W, \beta)$ comes from an absolutely irreducible representation of one absolutely simple factor of $G$. He also showed [19, Thm. 2] that this additional condition holds when $G_{\mathbf{R}}$ has no compact factors, if $(\sigma, W)$ is assumed to be primary over $\mathbf{Q}$ to begin with. However, if $(\sigma, W)$ is any representation of $G$ over $\mathbf{Q}$ admitting a symplectic structure (with which it satisfies $\left(H_{1}\right)$ ), then it has a decomposition into Q-primary components each of which itself admits a symplectic structure (with which it satisfies $\left(H_{1}\right)$ ) $[\mathbf{2 0}, \S 2.1]$. So it suffices to consider only Q-primary representations $(\sigma, W)$. On the other hand, as far as condition $\left(H_{1}\right)$ is concerned, we may assume without loss of generality that $G$ is simply connected as an algebraic group. Furthermore, under the assumption that $(\sigma, W)$ comes from an absolutely irreducible representation of one absolutely simple factor of $G$, it is enough to consider only $\mathbf{Q}$-simple $G$, for the extra assumption implies that $\sigma$ is nontrivial on only one Q-simple factor of $G$ [20, §7.1]. With these assumptions, then, Satake concludes $[\mathbf{2 0}, \S 8.1]$ that the $\mathbf{Q}$-simple algebraic groups possessing a symplectic representation satisfying condition $\left(\mathrm{H}_{1}\right)$ are of the form $G=R_{F / Q}\left(G_{1}\right)$ for some totally real algebraic number field $F$ and some absolutely simple, simply connected algebraic group $G_{1}$ over $F$ of type (I), (II), (III.1), (III.2), (IV.1) or (IV.2).

As for what Q-primary symplectic representations of these groups satisfy the above assumptions as well as condition $\left(H_{1}\right)$, Satake shows [20, $\S 8.3]$ that in addition to the "standard solutions" in the first four cases, where $(\sigma, W)$ is equivalent to some number of copies of $R_{F / Q}\left(\rho_{1}\right)$, with $\rho_{1}$ equivalent over $F$ to the identity representation of $G_{1}$, there are also "nonstandard solutions" for the groups of type (IV.1), (IV.2) and (I'), and these are all. For further details, see [20].

B. Some privileged representations. The next problem is to look for Q-privileged representations of the groups on Satake's list which are contained in the exterior powers of symplectic representations satisfying condition $\left(h_{1}\right)$. In this section we will restrict attention to symplectic representations $(\sigma, W, \beta)$ such that the linear closure of $\sigma\left(G_{\mathbf{R}}\right)$ in $\operatorname{End}\left(W_{\mathbf{R}}\right)$ contains the space $\tau(\mathfrak{X})$ of complex structures on $W_{\mathbf{R}}$. For under this hypotheses, which is satisfied when $G_{\mathbf{R}}$ has no compact factors $[13, \S \mathrm{F}]$, $C_{1}(G, W)$ is precisely the commutator algebra of $\sigma(G)$ in $\operatorname{End}(W)$. Then using classical invariant theory, when $G$ is a $\mathbf{Q}$-form of a classical group we have the following result. 
Proposition. Let $G$ be a simply connected semisimple algebraic group, simple over $\mathbf{Q}$, of the form $G=R_{F / \mathbf{Q}}\left(G_{1}\right)$ for some totally real algebraic number field $F$ and some simply connected absolutely simple algebraic group $G_{1}$ over $F$ of type (I), (III.1) or (III.2) such that $G_{\mathbf{R}}$ has no compact factors. Then every $\mathbf{Q}$-primary representation of $G$ is privileged over $\mathbf{Q}$.

Proof. We give the proof for $G$ of type (III), as the proof in case $G$ is of type (I) is quite similar once the conjugate-identity representations are taken into account. Let $\sigma=R_{F / \mathbf{Q}}\left(\rho_{1}^{\prime}\right)$, where $\rho_{1}^{\prime}$ is equivalent to the identity representation of $G_{1}$ over $F$. We will show that for a given Q-primary representation $\pi$ of $G$ there exist positive integers $m$ and $b$ such that $\pi$ is (equivalent to) a privileged constituent of $\Lambda^{b}(m \sigma)$ over $\mathbf{Q}$.

To begin with, we claim that it suffices to show that an absolutely irreducible component $\rho$ of $\pi$ is a privileged constituent of $\Lambda^{b}(m \sigma)$ (over some field) for some $b$ and $m$. For by $[\mathbf{2 0}, \S 1.1]$ we may assume that

$$
\pi \sim \bigoplus_{i=1}^{l}(k \rho)^{\tau_{l}},
$$

where $\rho^{\prime}:=k \rho$ is an absolutely primary component of $\pi$ and $\left\{\tau_{1}, \ldots, \tau_{l}\right\}$ is a set of representations for $\operatorname{Gal}(\overline{\mathbf{Q}} / \mathbf{Q}) /\left\{\tau \in \operatorname{Gal}(\overline{\mathbf{Q}} / \mathbf{Q}) \mid\left(\rho^{\prime}\right)^{\tau} \sim \rho^{\prime}\right\}$. Now if $\rho$ is a privileged constituent of $\Lambda^{b}(m \sigma)$, then $\rho^{\prime}$ is a privileged constituent of $\Lambda^{b}(k m \sigma)$, for this latter contains $k \Lambda^{b}(m \sigma)$ as a privileged constituent by Lemma 1B. Likewise $\left(\rho^{\prime}\right)^{\tau_{t}}$ is a privileged constituent of $\left(\wedge^{b}(k m \sigma)\right)^{\tau_{l}}$, which equals $\Lambda^{b}(k m \sigma)$ since $\sigma$ is defined over $\mathbf{Q}$. Using Lemma $1 \mathrm{~B}$ again, we therefore conclude that $\pi$ is a privileged constituent of $\wedge^{b}(k \operatorname{lm} \sigma)$.

Thus it remains to show that an absolutely irreducible component $\rho$ of $\pi$ is privileged. Let $[F: \mathbf{Q}]=: s$; then over a sufficiently large field, $G \simeq G_{1} \times \cdots \times G_{s}$, where each $G_{i}$ is an absolutely simple algebraic group Galois-conjugate to $G_{1}$, with $G_{1}$ defined over $F$. Furthermore $\sigma \sim$ $r\left(\oplus_{i=1}^{s} \sigma_{i}\right)$, where $\sigma_{i}=\sigma_{l}^{\prime} \circ p_{l}$ with $\sigma_{l}^{\prime}$ an absolutely simple representation of $G_{l}$ equivalent to the identity representation, $p_{l}: G \rightarrow G_{l}$ the projection map, and $r$ a positive integer (the square root of the dimension of the division algebra over $F$ which enters into the definition of $G_{1}$ ). Then $\rho$, being absolutely irreducible, will be of the form $\rho \sim \rho_{1} \circ p_{1} \otimes \cdots \otimes \rho_{s} \circ \rho_{s}$, for some absolutely irreducible representations $\rho_{i}$ of $G_{i}$, for $1 \leq i \leq s$. On the other hand, for any $s$-tuple $\left(b_{1}, \ldots, b_{s}\right)$ of natural numbers such that $\sum b_{\imath}=b$, the representation $\Lambda^{b}(m \sigma) \sim \Lambda^{b}\left(m r\left(\oplus_{i=1}^{s} \sigma_{i}\right)\right)$ contains a privileged constituent of the form $\wedge^{b_{1}}\left(m r \sigma_{1}\right) \otimes \cdots \otimes \bigwedge^{b_{s}}\left(m r \sigma_{s}\right)$, which in turn contains a privileged constituent of the form $\sigma_{1}^{\otimes b_{1}} \otimes \cdots \otimes \sigma_{s}^{\otimes b_{s}}$ whenever 
$b_{i} \leq m r$ for all $1 \leq i \leq s$. Here we have repeatedly used Lemma $1 \mathrm{~B}$ and the observation that if two $G$-submodules $U$ and $V$ of some $G$-module contain only $\{0\}$ in common then $U \wedge V$ and $U \otimes V$ are isomorphic as vector spaces and $G$-modules. Now the main theorem of classical invariant theory (cf. [22]) asserts that if $V^{\prime}$ is a standard (identity) representation of a classical group $G^{\prime}$, then $\operatorname{End}_{G}\left(V^{\prime \otimes n}\right)$ is generated by the natural image therein of $\operatorname{End}_{G}\left(V^{\prime} \times \cdots \times V^{\prime}\right)$. Applying this to each $\sigma_{i}^{\otimes b_{i}}$ and taking the product over $1 \leq i \leq s$, it follows that $\operatorname{End}_{G}(V)$, where $V$ denotes the space of $\sigma_{1}^{\otimes b_{1}} \otimes \cdots \otimes \sigma_{s}^{\otimes b_{s}}$, is generated by the restrictions to $V$ of the elements of $C_{b}(G, W)$, where $W$ is the space of $m \sigma$. Since $\sigma_{1}^{\otimes b_{1}}$ $\otimes \cdots \otimes \sigma_{s}^{\otimes b_{s}}$ is privileged, it follows from this that every constituent of $\sigma_{1}^{\otimes b_{1}} \otimes \cdots \otimes \sigma_{s}^{\otimes b_{s}}$ is also privileged. In particular, by choosing $\left(b_{1}, \ldots, b_{s}\right)$ appropriately and $m$ sufficiently large, the given representation $\rho$ is privileged, as required.

COROLlaRY. Let $G$ be a simply connected semisimple algebraic group over $\mathbf{Q}$, simple over $\mathbf{Q}$ and of $\mathbf{Q}$-rank zero, having the form $G=R_{F / \mathbf{Q}}\left(G_{1}\right)$ for some totally real algebraic number field $F$ and some simply connected absolutely simple algebraic group $G_{1}$ over $F$ of type (I) or (III.2) such that $G_{\mathbf{R}}$ has no compact factors, and let $(\pi, E)$ be any $\mathbf{Q}$-primary representation of $G$. Then for any torsion-free arithmetic subgroup $\Gamma$ of $G$ there exists a Kuga fiber variety $\mathscr{V}$ such that $H^{a}(\Gamma ; E)$ may be identified with an algebraically defined subspace of $H^{*}(\mathscr{V} ; \mathbf{Q})$.

EXAMPLE OF TYPE (III.2). Suppose $G_{\mathbf{Q}}$ is the group of units of norm 1 in a totally indefinite quaternion algebra $B$ over a totally real number field $F$ of degree $s$ over $\mathbf{Q}$. Let $\mathcal{O}$ be an order in $B$, and $\Gamma$ a torsion-free subgroup of finite index in the group of norm 1 units of $\mathcal{O}$. Also let $\sigma$ denote the representation of $G$ given by left multiplication on $B$. Then for each $m \geq 1$, one may put a symplectic structure $\beta$ on $B^{m}:=B \times \cdots \times B$ ( $m$ times) which takes integral values on $\mathcal{O}^{m} \times \mathcal{O}^{m}$. Therefore the quotient $\mathscr{V}:=\Gamma \backslash \mathfrak{X} \times\left(\left(B^{m} \otimes_{\mathbf{Q}} \mathbf{R}\right) / \mathcal{O}^{m}\right)$ can be given the structure of a Kuga fiber variety where $\mathfrak{X}$, the symmetric domain associated to $G$, is isomorphic to a product of $s$ complex upper half-planes. Over a sufficiently large field $G \simeq \mathrm{SL}_{2} \times \cdots \times \mathrm{SL}_{2}$, and $\sigma \sim 2 \oplus_{i=1}^{s} \sigma_{i}$, where each $\sigma_{i}$ is 2-dimensional and all factors of $\mathrm{SL}_{2}$ except the $i$ th are in the kernel of $\sigma_{i}$.

We claim that every representation-theoretic component of $H^{*}(\mathscr{V} ; \mathbf{Q})$ is algebraically defined. To prove this, it suffices to show that every constituent of $\wedge^{b}(m \sigma)$ is privileged, for any $b$ with $0 \leq b \leq 4 m s$. As 
before

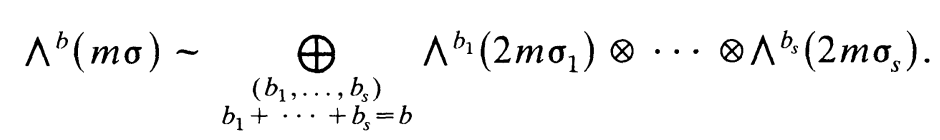

Let $\rho_{1}$ denote the standard 2-dimensional representation of $\mathrm{SL}_{2}$. Then the problem is reduced to showing that the commutator algebra of $\left(\bigwedge^{c}\left(2 m \rho_{1}\right)\right)\left(\mathrm{SL}_{2}\right)$ in the endomorphisms of the space of that representation is generated by the image therein of the commutator algebra of $\mathrm{SL}_{2}$ acting diagonally on $2 m$ copies of (the space of) $\rho_{1}$. However, as in Lemma $1 \mathrm{~B}$, as an $\mathrm{SL}_{2}$-module $\Lambda^{c}\left(2 m \rho_{1}\right)$ is a sum of pieces of the form $\Lambda^{c_{1}} \rho_{1} \otimes \cdots \otimes \Lambda^{c} 2 m \rho_{1}$, where $c_{1}+\cdots+c_{2 m}=c$ with $0 \leq c_{J} \leq 2$. Each of these pieces in turn is isomorphic, as an $\mathrm{SL}_{2}$-module, to $\rho_{1}^{\otimes c^{\prime}}$, where $c^{\prime}$ is the number of 1's occurring among $\left(c_{1}, \ldots, c_{2 m}\right)$. But, as is well-known [22], the commutator algebra of $\mathrm{SL}_{2}$ in the endomorphisms of the space of $\rho_{1}^{\otimes c^{\prime}}$ is generated by permutations of the factors. Since all these permutations are induced by permuting $2 m$ irreducible factors of $2 m \rho_{1}$, every constituent of $\rho_{1}^{\otimes c^{\prime}}$ is privileged and the claim is established.

C. A remark on algebraic cycles. In [16, §1] Millson describes a very general method of constructing "higher weight cycles" in torus bundles over locally symmetric spaces. If $\iota$ is an element of order 2 in $G_{\mathbf{Q}}$, then it induces an involution on the symmetric space $\mathfrak{X}$ whose fixed points are denoted $\mathfrak{X}_{\iota}$. The image $\mathscr{C}_{\iota}$ of $\mathfrak{X}_{\iota}$ in $\mathscr{U}=\Gamma \backslash \mathfrak{X}$ is called a special cycle. Let $\Gamma_{\iota}$ denote the $\iota$-invariant elements of $\Gamma$. Then when $V$ is a $\Gamma_{\iota}$-stable oriented rational subspace of $W$ with a $\Gamma_{\iota}$-invariant orientation, the image $T(\iota, V)$ of $\mathfrak{X}_{\iota} \times V_{\mathbf{R}}$ in $\mathscr{V}:=\Gamma \backslash \mathfrak{X} \times\left(W_{\mathbf{Q}} / \Lambda\right)$ is a special cycle of higher weight. Moreover, if $\mathfrak{X}$, has codimension $a$ in $\mathfrak{X}$ and $V$ has codimension $b$ in $W$, then the fundamental class $\operatorname{cl}[T(\iota, V)]$ of $T(\iota, V)$ in $H^{*}(\mathscr{V} ; \mathbf{Q})$ will in fact lie in $H^{\langle a, b\rangle}(\mathscr{V} ; \mathbf{Q})$ [16, Thm. 1.1]. Now suppose $G, \Gamma, W$, etc. satisfy all the conditions for $\mathscr{V}$ to have the structure of a complex projective variety. Then whenever $\mathfrak{X}_{\iota}$ is a hermitian domain holomorphically embedded in $\mathfrak{X}$, and $V$ is $J_{x}$-stable for all $x \in \mathfrak{X}$, it follows that $T(\iota, V)$ is a subvariety of $\mathscr{V}$. Therefore the component of $\mathrm{cl}[T(\iota, V)]$ in any representation-theoretic component of $H^{\langle a, b\rangle}(\mathscr{V} ; \mathbf{Q})$ is an algebraic class in that component.

Subvarieties of the form $T(\iota, V)$ were considered in [5] (see also [4]) in the special case where $G$ was a $\mathbf{Q}$-form of $\mathrm{SL}_{2}(\mathbf{R}) \times \mathrm{SL}_{2}(\mathbf{R})$ of Q-rank zero. There a Kuga fiber variety $\mathscr{V}$ was constructed whose middle cohomology $H^{4 k+2}(\mathscr{V} ; \mathbf{Q})$ contained an (unique) algebraically defined representation-theoretic component $H^{4 k+2}(\mathscr{M} ; \mathbf{Q})$ corresponding to 
$H^{2}(\Gamma ; E)$, where $E$ is the space of $\rho_{2 k} \circ p_{1} \otimes \rho_{2 k} \circ p_{2}$, with $\rho_{2 k}$ being the symmetric tensor representation of $\mathrm{SL}_{2}$ of degree $2 k$ and $p_{i}$ being the projection onto the $i$ th factor for $i=1,2$. Then a suitable collection of $T(\iota, V)$ were constructed and a special case of Theorem 1 implied that the components in $H^{4 k+2}(\mathscr{M} ; \mathbf{Q})$ of the fundamental classes cl[ $\left.T(\iota, V)\right]$ of the $T(\iota, V)$ were algebraic. Then harmonic differential $(2 k+1,2 k+1)$ forms on $\mathscr{V}$ representing these classes in $H^{4 k+2}(\mathscr{M} ; \mathbf{Q})$ were constructed, their pairwise intersection multiplicities (cup products) were computed, and it was shown, in the manner of Hirzebruch and Zagier [8] and Kudla [11], that the generating function for these intersection numbers was a modular form of higher weight, cf. [5] for more details.

\section{REFERENCES}

[1] A. Borel, Density and maximality of arithmetic groups, J. Reine Angew. Math., 224 (1966), 78-89.

[2] A. Borel and Harish-Chandra, Arithmetic subgroups of algebraic groups, Ann. of Math., 75 (1962), 485-535.

[3] P. Deligne, Valeurs de fonctions $L$ et périods d'intégrales, in Automorphic Forms, Representations and L-functions, Part 2, pp. 313-346; Amer. Math. Soc., Proc. Symp. Pure Math., 33 (1979),

[4] B. B. Gordon, Intersections of higher weight cycles over quaternionic modular surfaces and modular forms of Nebentypus, Bull. Amer. Math. Soc., 14 (1986), 293-298.

[5] - Intersections of higher weight cycles and modular forms, to appear.

[6] P. Griffiths and J. Harris, Principles of Algebraic Geometry, John Wiley \& Sons, New York etc., 1978.

[7] A. Grothendieck, Standard conjectures on algebraic cycles, in Algebraic Geometry: Papers Presented at the Bombay Colloquium, 1969, Oxford Univ. Press, Oxford etc., 1969.

[8] F. Hirzebruch and D. Zagier, Intersection numbers of curves on Hilbert modular surfaces and modular forms of Nebentypus, Invent. Math., 36 (1976), 57-113.

[9] S. L. Kleiman, Motives, in Algebraic Geometry, Oslo, 1970. Proc. Fifth Nordic Summer School in Math., Oslo, 1970, Wolters-Noordhoff, Groningen, 1972, pp. 53-82.

[10] K. Kodaira, On Kähler varieties of restricted type, Ann. of Math., 60 (1954), 28-48.

[11] S. S. Kudla, Intersection numbers of quotients of the compels 2-ball and Hilbert modular forms, Invent. Math., 47 (1978), 189-208.

[12] M. Kuga, Fibre Varieties over a Symmetric Space whose Fibres are Abelian Varieties, vols. I and II, Univ. of Chicago, Chicago, 1964.

[13] _ Fibre varieties over symmetric space whose fibres are abelian varieties, in Proc. of the U.S.-Japan Seminar in Differential Geometry, Kyoto, Japan, 1965, Nippon Hyoronsha, (1966), 72-81.

[14] _ Algebraic cycles in gtfabv, J. Fac. Sci. Univ. Tokyo, Sec. IA, Math., 29 (1982), 13-29.

[15] Yu. Manin, Correspondences, motifs and monoidal transformations, Mat. Sbornik, 77 (119), 475-507; Amer. Math. Soc. Transl., (1970).

[16] J. Millson, Higher weight cycles, J. Fac. Sci. Univ. Tokyo, sec. IA, Math., 28 (1981), 465-479. 
[17] G. D. Mostow and J. H. Sampson, Linear Algebra, McGraw-Hill, New York, etc., 1969.

[18] G. D. Mostow and T. Tamagawa, On the compactness of arithmetically defined homogeneous spaces, Ann. of Math., 76 (1962), 440-463.

[19] I. Satake, Holomorphic embeddings of symmetric domains into a Siegel space, Amer. J. Math., 87 (1965), 425-461.

[20] __ Symplectic representations of algebraic groups satisfying a certain analyticity condition, Acta Math., 117 (1967), 215-299.

[21] _ Algebraic Structures of Symmetric Domains, Princeton Univ. Press, Princeton and Iwanami Shoten, Japan, 1980.

[22] H. Weyl, The Classical Groups, Princeton Univ. Press, Princeton, 1946.

Received September 18, 1986 and in revised form January 19, 1987.

\section{PURDUE UNIVERSITY}

WEST LAFAYETTE, IN 47907

Current Address: Department of Mathematics

University of Oklahoma

Norman, Oklahoma 73019 


\section{PACIFIC JOURNAL OF MATHEMATICS EDITORS}

\author{
V. S. VARADARAJAN \\ (Managing Editor) \\ University of California \\ Los Angeles, CA 90024 \\ HERBERT ClEMENS \\ University of Utah \\ Salt Lake City, UT 84112 \\ R. FINN \\ Stanford University \\ Stanford, CA 94305
}

\author{
HERMANN FLASCHKA \\ University of Arizona \\ Tucson, AZ 85721
}

RAMESh A. GANGolli

University of Washington Seattle, WA 98195

VAUGHAN F. R. JONES

University of California

Berkeley, CA 94720

\author{
ROBION KIRBY \\ University of California \\ Berkeley, CA 94720
}

C. C. MOORE

University of California

Berkeley, CA 94720

HAROLD STARK

University of California, San Diego

La Jolla, CA 92093

\section{ASSOCIATE EDITORS}
R. AREnS
E. F. BECKENBACH
B. H. NEUMANN
F. WOLF
K. YOSHIDA

(1906-1982)

\section{SUPPORTING INSTITUTIONS}

\begin{abstract}
UNIVERSITY OF ARIZONA
UNIVERSITY OF BRITISH COLUMBIA

CALIFORNIA INSTITUTE OF TECHNOLOGY

UNIVERSITY OF CALIFORNIA

MONTANA STATE UNIVERSITY

UNIVERSITY OF NEVADA, RENO

NEW MEXICO STATE UNIVERSITY

OREGON STATE UNIVERSITY
\end{abstract}

\author{
UNIVERSITY OF OREGON \\ UNIVERSITY OF SOUTHERN CALIFORNIA \\ STANFORD UNIVERSITY \\ UNIVERSITY OF HAWAII \\ UNIVERSITY OF TOKYO \\ UNIVERSITY OF UTAH \\ WASHINGTON STATE UNIVERSITY \\ UNIVERSITY OF WASHINGTON
}

The Supporting Institutions listed above contribute to the cost of publication of this Journal, but they are not owners or publishers and have no responsibility for its content or policies.

Mathematical papers intended for publication in the Pacific Journal of Mathematics should be in typed form or offset-reproduced (not dittoed), double spaced with large margins. Please do not use built up fractions in the text of the manuscript. However, you may use them in the displayed equations. Underline Greek letters in red, German in green, and script in blue. The first paragraph must be capable of being used separately as a synopsis of the entire paper. In particular it should contain no bibliographic references. Please propose a heading for the odd numbered pages of less than 35 characters. Manuscripts, in triplicate, may be sent to any one of the editors. Please classify according to the scheme of Math. Reviews, Index to Vol. 39. Supply name and address of author to whom proofs should be sent. All other communications should be addressed to the managing editor, or Elaine Barth, University of California, Los Angeles, California 90024.

There are page-charges associated with articles appearing in the Pacific Journal of Mathematics. These charges are expected to be paid by the author's University, Government Agency or Company. If the author or authors do not have access to such Institutional support these charges are waived. Single authors will receive $\mathbf{5 0}$ free reprints; joint authors will receive a total of $\mathbf{1 0 0}$ free reprints. Additional copies may be obtained at cost in multiples of 50 .

The Pacific Journal of Mathematics is issued monthly as of January 1966. Regular subscription rate: $\$ 190.00$ a year (5 Vols., 10 issues). Special rate: $\$ 95.00$ a year to individual members of supporting institutions.

Subscriptions, orders for numbers issued in the last three calendar years, and changes of address should be sent to Pacific Journal of Mathematics, P.O. Box 969, Carmel Valley, CA 93924, U.S.A. Old back numbers obtainable from Kraus Periodicals Co., Route 100, Millwood, NY 10546.

The Pacific Journal of Mathematics at P.O. Box 969, Carmel Valley, CA 93924 (ISSN 0030-8730) publishes 5 volumes per year. Application to mail at Second-class postage rates is pending at Carmel Valley, California, and additional mailing offices. Postmaster: send address changes to Pacific Journal of Mathematics, P.O. Box 969, Carmel Valley, CA 93924.

PUBLISHED BY PACIFIC JOURNAL OF MATHEMATICS, A NON-PROFIT CORPORATION Copyright (C) 1988 by Pacific Journal of Mathematics 


\section{Pacific Journal of Mathematics}

\section{Vol. 131, No. 2 December, 1988}

Selman Akbulut and Henry Churchill King, Polynomial equations of immersed surfaces .................................... 209

Alberto Baider and Richard C. Churchill, The Campbell-Hausdorff group and a polar decomposition of graded algebra automorphisms ........2 219

Wayne C. Bell and John William Hagood, Separation properties and exact

Radon-Nikodým derivatives for bounded finitely additive measures . . . 237

Dennis J. Garity, James P. Henderson and David G. Wright, Menger

spaces and inverse limits ...............................249

B. Brent Gordon, Algebraically defined subspaces in the cohomology of a

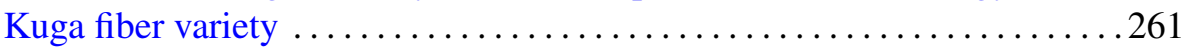

Jeffrey A. Hogan, Weighted norm inequalities for the Fourier transform on connected locally compact groups ........................... 277

Guojun Liao, A study of regularity problem of harmonic maps ..........291

Chin-pi Lu, Modules satisfying ACC on a certain type of colons ......... 303

Kunio Murasugi, Jones polynomials of periodic links

Hans Schoutens, Approximation properties for some non-Noetherian local

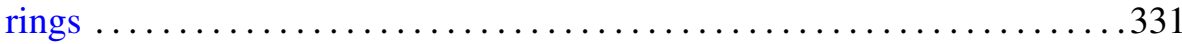

Peter Sjögren, Convergence for the square root of the Poisson kernel ...... 361 Alexandru Ion Suciu, The oriented homotopy type of spun 3-manifolds .393 